\title{
On direct one-neutron decay of the giant dipole resonance
}

\author{
Boris Tulupov ${ }^{1}$ and Mikhail Urin ${ }^{2, *}$ \\ ${ }^{1}$ Institute for Nuclear Research RAS, Moscow, Russia \\ ${ }^{2}$ National Research Nuclear University "MEPhI” (Moscow Engineering Physics Institute), Moscow, Russia
}

\begin{abstract}
The newly developed particle-hole dispersive optical model is implemented to evaluate the partial and total branching ratios for direct one-neutron decay of the giant dipole resonance in a few neutron-closedshell nuclei. The calculation results obtained for ${ }^{48} \mathrm{Ca},{ }^{90} \mathrm{Zr},{ }^{140} \mathrm{Ce}$ and ${ }^{208} \mathrm{~Pb}$ with no specific model parameters are compared with available experimental data.
\end{abstract}

\section{Introduction}

Experimental and theoretical studies of direct one-nucleon decays of any giant resonance carry information about the microscopic structure and decay mechanisms of highenergy particle-hole-type nuclear excitations. In the present work, we implement the newly developed particlehole dispersive optical model (PHDOM) [1] to describe direct one-neutron decay of the isovector giant dipole resonance in the neutral channel (abbreviated below as GDR) for neutron-closed-shell nuclei. The first attempt of such a description has been undertaken in Ref. [2] for ${ }^{208} \mathrm{~Pb}$. Being somewhat improved recently [3], the method of Refs. $[1,2]$ is used in the present work to describe partial and total branching ratios for direct one-neutron decay of the GDR in ${ }^{48} \mathrm{Ca},{ }^{90} \mathrm{Zr},{ }^{140} \mathrm{Ce}$ and ${ }^{208} \mathrm{~Pb}$. The method consists in evaluation within the PHDOM of the energyaveraged isovector dipole strength function and partial one-neutron-escape strength functions, which determine, respectively, the $E 1$ photoabsorption cross section and the cross sections of the partial "direct + semi-direct" $E 1(\gamma, n)$ reaction accompanied by population of a certain one-hole state of the product nucleus. Then the partial branching ratio is defined as the ratio of the mentioned one-neutronescape strength function integrated over a GDR region to the isovector dipole strength function integrated over the same region. If this region is limited by the threshold of two-neutron emission, the total direct-decay branching ratio is, actually, the ratio of the direct to total (i.e., direct + statistical) one-neutron decay probabilities for the considered GDR.

In Sec. 2, we briefly describe the calculation scheme, using, as a base, Refs. [2, 3]. Input quantities, choice of model parameters and calculation results are given in Sec. 3. Section 4 contains the summary and perspectives of further studies

\footnotetext{
*e-mail: urin@theor.mephi.ru
}

\section{Calculation scheme}

The PHDOM is the microscopically-based extension of the standard [4] and nonstandard [5] continuum-RPA (cRPA) versions to a phenomenological (and averaged over an energy) description of the spreading effect. For this reason, the basic PHDOM equations remind the corresponding cRPA equations. The difference consists in using the "free" p-h propagator in evaluation of various energy-averaged strength functions, and also the opticalmodel like continuum-state wave functions for the escaped (captured) nucleons in evaluation of the energy-averaged "direct + semi-direct" (DSD) reaction amplitudes [1-3]. These quantities are evaluated with taking the spreading effect into account in terms of the properly parameterized energy-averaged $p-h$ self-energy term. The imaginary part of this term determines the real part via the dispersive relationship, which follows from the spectral expansion of the $2 p-2 h$ Green function $(2 p-2 h$ configurations are the doorway states for the spreading effect).

For medium-heavy nuclei, having a relatively small neutron excess, the E1-photoabsorption cross section $\sigma_{\alpha, E 1}(\omega)$ is determined by the isovector dipole strength function $S_{1}^{(-)}(\omega)$ related to the single-particle external field $V_{0,1}^{(-)}=r Y_{1 M}$ (hereafter, we use the notations of Ref. [2]).

$$
\begin{gathered}
\sigma_{\alpha, E 1}(\omega)=C \omega S_{1}^{(-)}(\omega) \\
S_{1}^{(-)}(\omega)=-\frac{1}{2 \pi} \operatorname{Im} \int \mathrm{r} \mathcal{A}_{1}\left(\mathrm{r}, \mathrm{r}^{\prime}, \omega\right) \mathrm{V}_{1}^{(-)}\left(\mathrm{r}^{\prime}, \omega\right) \mathrm{drdr}^{\prime}
\end{gathered}
$$

Here, $\left(r r^{\prime}\right)^{(-2)} \mathcal{A}_{1}\left(r, r^{\prime}, \omega\right)$ is the radial part of the "free" $\mathrm{p}$-h propagator, corresponding to the excitations in the neutral channel with angular momentum $L=1$ (see, e.g., Refs. [1,2]), $\omega$ is the photon energy and $C=\frac{16 \pi^{2}}{3} \frac{e^{2}}{h c}$. The cross section of the DSD partial $E 1(\gamma, n)$ reaction accompanied by population of neutron-hole state $\mu^{-1}$ in the product nucleus, $\sigma_{\mu, E 1}$, is determined by the respective squared 
partial DSD-reaction amplitudes [3]

$$
\sigma_{\mu, E 1}=C \omega \sum_{(\lambda)}\left|M_{1, c}^{(n)}(\omega)\right|^{2} \equiv C S_{\mu, 1}^{(n), \uparrow}(\omega) .
$$

In accordance with Ref. [3], the below-given expression for the squared partial amplitude only slightly differs from the respective relationship in Ref. [2]

$$
\begin{aligned}
& \left|M_{1, c}^{(n)}(\omega)\right|^{2}= \\
& \quad \frac{1}{4} n_{\mu}\left(t_{(\lambda)(\mu)}^{(1)}\right)^{2}\left|\int \chi_{\varepsilon,(\lambda)}^{*}(r) V_{1}^{(-)}(r, \omega) \chi_{\mu}(r) d r\right|^{2}
\end{aligned}
$$

Here, $c=\mu,(\lambda)$ is the set of the reaction-channel quantum numbers, that includes the neutron bound-state quantum numbers $\mu$ and the escaped-neutron quantum numbers $(\lambda)=j_{\lambda}, l_{\lambda} ; n_{\mu}$ is the occupation factor (equal to unity for the neutron-closed-shell target nuclei); $\square 3 t_{(\lambda)(\mu)}^{(1)}=$ $\left\langle(\lambda)\left\|Y_{1}\right\|(\mu)\right\rangle$ is the reduced matrix element; $\left.r^{(}-1\right) \chi_{\varepsilon,(\lambda)}(r)$ is the optical-model like neutron continuum-state wave function, corresponding to the kinetic energy $\varepsilon=\varepsilon_{\mu}+\omega$ and having the standing-wave asymptotic behavior (in the cRPA limit, i.e. in ignoring the spreading effect, these wave functions are normalized to $\delta$-function of the energy); $\left.r^{(}-1\right) \chi_{\mu}(r)$ is the neutron bound-state wave function; $V_{1}^{(-)}(r, \omega)$ is the radial effective field related to the aforementioned external field and obeying the integral equation, which contains the $p-h$ interaction and "free" $p-h$ propagator [2].

The partial branching ratio for direct one-neutron decay of the GDR, $b_{\mu, E 1}^{(n)}(\delta)$, can be defined via the strength functions of Eqs. (1)-(3)

$$
b_{\mu, E 1}^{(n)}(\delta)=\int_{(\delta)} S_{\mu, 1}^{(n), \uparrow} / \int_{(\delta)} S_{1}^{(-)}(\omega) d \omega
$$

where $\delta$ is the excitation-energy interval in a vicinity of the given GDR. If this interval is limited by the threshold of two-neutron emission, the total direct one-neutron decay branching ratio, $b_{E 1}^{(n), t o t}(\delta)=\sum_{\mu} b_{E 1}^{(n)}(\delta)$, is the ratio of direct to total ("direct + statistical") one-neutron GDR decay probabilities.

\section{Input quantities. Calculation results}

In implementations of the PHDOM, the following input quantities are currently used [2,6]: (i) a phenomenological $p-h$ interaction responsible for long-range correlations; (ii)a phenomenological mean field partially consistent with this interaction; (iii) the imaginary part of the properly parameterized energy-averaged $p-h$ self-energy term responsible for the spreading effect. In the description of the aforementioned $E 1$ photo-nuclear reactions, the isovector part of the Landau-Migdal $p-h$ interaction (with the dimensionless strength $f^{\prime}$ ) and "velocity-dependent" forces (separable, with the dimensionless strength $k_{1}^{\prime}$ ) are exploited [2]. The phenomenological (Woods-Saxontype) mean field, in which the symmetry potential (proportional to $f^{\prime}$ ) and mean Coulomb field are calculated selfconsistently, is described in Refs. [2, 7].
Table 1. List of mean-field parameters used in calculations. For each nucleus $r_{0}$ is taken equal to $1.21 \mathrm{fm}$

\begin{tabular}{|c|c|c|c|c|}
\hline & $U_{0}, \mathrm{MeV}$ & $f^{\prime}$ & $U_{l s}, \mathrm{MeV} \cdot \mathrm{fm}^{2}$ & $a, \mathrm{fm}$ \\
\hline${ }^{48} \mathrm{Ca}$ & 54.61 & 1.16 & 32.20 & 0.60 \\
\hline${ }^{90} \mathrm{Zr}$ & 55.21 & 1.05 & 31.88 & 0.61 \\
\hline${ }^{140} \mathrm{Ce}$ & 55.82 & 0.98 & 32.09 & 0.62 \\
\hline${ }^{208} \mathrm{~Pb}$ & 56.39 & 0.98 & 33.35 & 0.63 \\
\hline
\end{tabular}

Table 2. Values of the adjustable parameters determining the strengths of the imaginary part of self-energy term and the isovector part of "velocity dependent" forces.

\begin{tabular}{|c|c|c|c|c|}
\hline & ${ }^{48} \mathrm{Ca}$ & ${ }^{90} \mathrm{Zr}$ & ${ }^{140} \mathrm{Ce}$ & ${ }^{208} \mathrm{~Pb}$ \\
\hline$\alpha, \mathrm{MeV}^{-1}$ & 0.10 & 0.12 & 0.10 & 0.08 \\
\hline$k_{1}^{\prime}$ & 0.27 & 0.15 & 0.13 & 0.17 \\
\hline
\end{tabular}

The mean-field strength $U_{0}, U_{l s}, f^{\prime}$ and geometrical parameters $\left(r_{0}, a\right)$ are found from the description of the observable quasi-particle spectra in doubly-closed-shell nuclei ${ }^{48} \mathrm{Ca},{ }^{132} \mathrm{Sn},{ }^{208} \mathrm{~Pb}$. For other nuclei these parameters are found using an interpolation procedure. The list of the mean field parameters for nuclei considered in this work is given in Table 1. The imaginary part of the strength of the aforementioned $p-h$ self-energy term is parameterized by a three-parametric function, exhibiting a saturationlike excitation energy dependence $[1,2]$. The "gap" and "saturation" parameters are chosen as universal quantities, while the strength parameter $\alpha$ (together with the strength $k_{1}^{\prime}$ ) is adjusted to reproduce in calculations within the PHDOM the energy and total width of the GDR in the experimental photo-absorption cross section. Then, the partial DSD $(\gamma, n)$ reaction cross section (and, therefore, the corresponding branching ratio) can be evaluated with no additional adjustable parameters.

A quality of the description within the current $\mathrm{PH}$ DOM version of the $E 1$ photo-absorption cross section in a vicinity of the GDR in the considered nuclei can be seen in Fig. 1, where the respective experimental data are also shown. The obtained adjusted parameters $\alpha$ and $k_{1}^{\prime}$ are given in Table 2.

The partial and total $\operatorname{DSD}(\gamma, n)$ reaction cross sections calculated for ${ }^{48} \mathrm{Ca}$ in the same energy region are shown in Figs. 2 and 3, respectively. The partial and total branching ratios for direct one-neutron decay of the GDR in the considered nuclei are evaluated in accordance with Eq. (4). The results obtained for two energy intervals (the lesser interval corresponds to the threshold of two-neutron emission) are given in Tables ??. The total direct one-neutron decay branching ratio is decreased with increasing of $A$ number due to decreasing of the GDR energy and, therefore, the energy of directly escaped neutrons, while the imaginary part of the $p-h$ self-energy term strength taken at the GDR energy is changed slightly. The single experimental result concerned with direct one-neutron decay of the GDR has been obtained in Ref. [11], where the value $b_{E 1}^{(n), t o t}(\delta)=39 \pm 5 \%(\delta=11-25 \mathrm{MeV})$ was deduced from the ${ }^{48} \mathrm{Ca}\left(e, e^{\prime} n\right)$ reaction cross section.

The corresponding calculation result (Table 4) is in a reasonable agreement with the mentioned experimental 

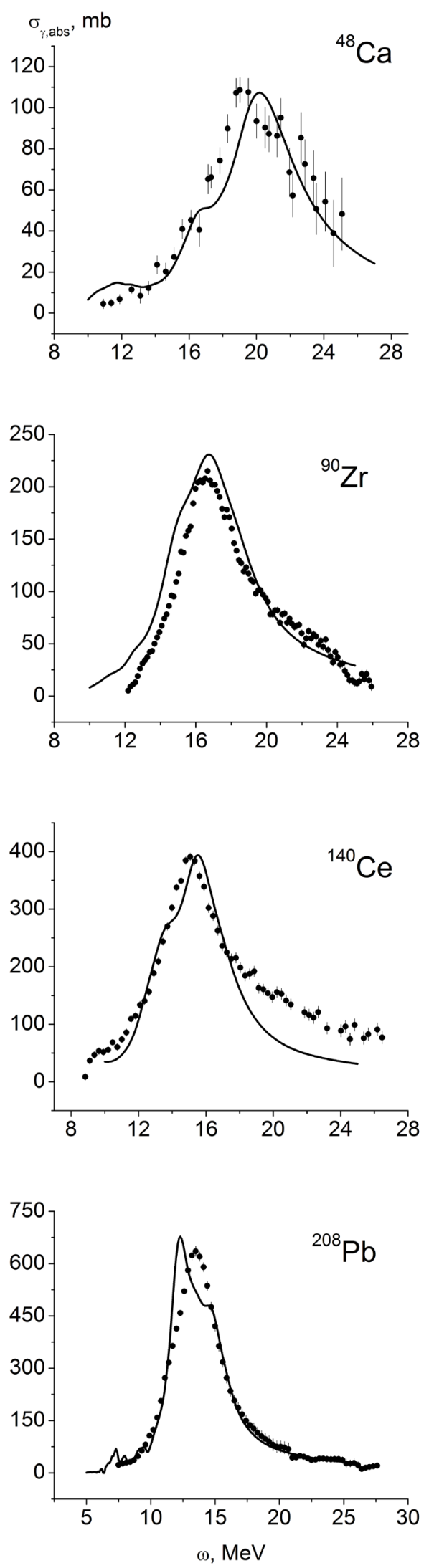

Figure 1. The $E 1$ photo-absorption cross sections calculated within the PHDOM in a vicinity of the GDR in ${ }^{48} \mathrm{Ca},{ }^{90} \mathrm{Zr},{ }^{140} \mathrm{Ce}$ and ${ }^{208} \mathrm{~Pb}$ (full lines). The respective adjustable parameters (see the text) are given in Table 2. The corresponding experimental data are taken from Refs. $[8]{ }^{48} \mathrm{Ca},[9]{ }^{90} \mathrm{Zr}$ and ${ }^{140} \mathrm{Ce},[10]{ }^{208} \mathrm{~Pb}$.

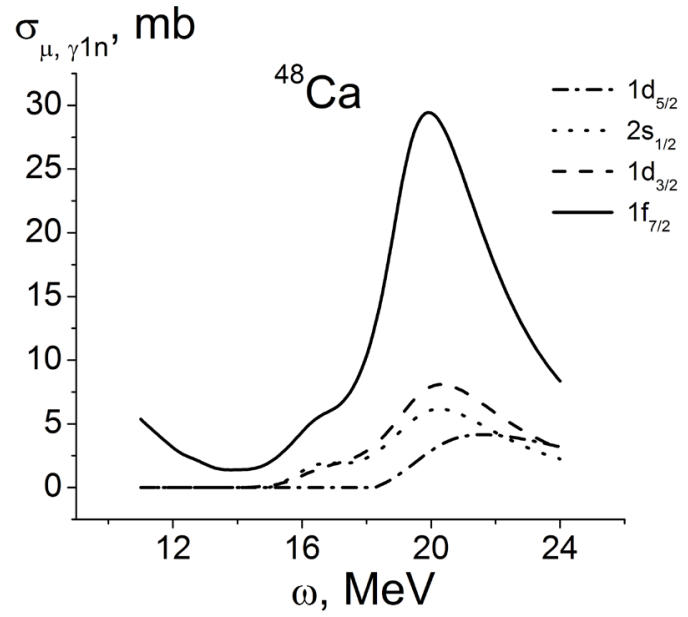

Figure 2. The partial DSD ${ }^{48} \mathrm{Ca}(\gamma, n)$ reaction cross sections calculated for the GDR energy region.

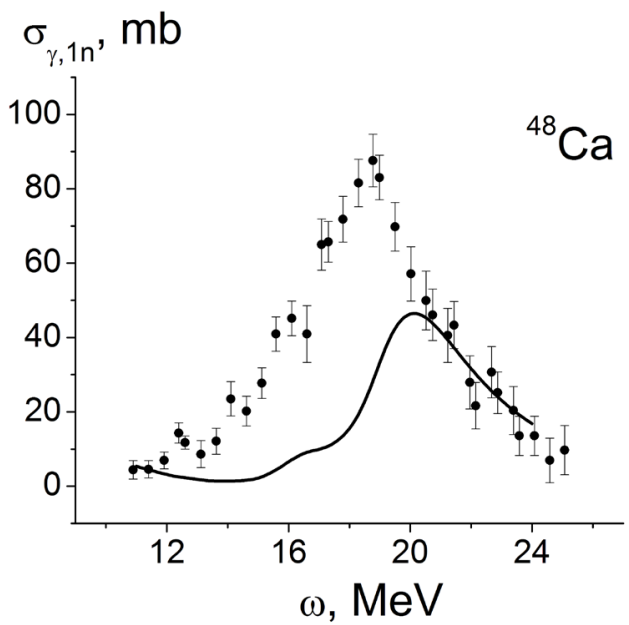

Figure 3. The calculated total DSD ${ }^{48} \mathrm{Ca}(\gamma, n)$ reaction cross section in a comparison with the experimental ${ }^{48} \mathrm{Ca}(\gamma, 1 n)$ reaction total cross section [8] for the GDR energy region.

Table 3. Total direct one-neutron decay branching ratios evaluated within the current PHDOM version for the GDR in ${ }^{48} \mathrm{Ca}$.

\begin{tabular}{|c|c|c|}
\hline$\mu$ & $b_{\mu, E 1}^{(n)}(\delta)$ & $b_{\mu, E 1}^{(n)}(\delta)$ \\
& $\delta=11-21 \mathrm{MeV}$ & $\delta=11-25 \mathrm{MeV}$ \\
\hline $1 \mathrm{~d}_{5 / 2}$ & $1.2 \%$ & $2.8 \%$ \\
\hline $2 \mathrm{~s}_{1 / 2}$ & $4.1 \%$ & $4.6 \%$ \\
\hline $1 \mathrm{~d}_{3 / 2}$ & $4.9 \%$ & $5.9 \%$ \\
\hline $1 \mathrm{f}_{7 / 2}$ & $21.2 \%$ & $22.0 \%$ \\
\hline
\end{tabular}

Table 4. Total direct one-neutron decay branching ratios evaluated within the current PHDOM version for the GDR in ${ }^{48} \mathrm{Ca},{ }^{90} \mathrm{Zr},{ }^{140} \mathrm{Ce}$ and ${ }^{208} \mathrm{~Pb}$ nuclei.

\begin{tabular}{|c|c|c|c|c|}
\hline & ${ }^{48} \mathrm{Ca}$ & ${ }^{90} \mathrm{Zr}$ & ${ }^{140} \mathrm{Ce}$ & ${ }^{208} \mathrm{~Pb}$ \\
\hline$\delta, \mathrm{MeV}$ & $11-25$ & $10-22$ & $7-16$ & $7-16$ \\
\hline$b_{E 1}^{(n), \text { tot }}, \%$ & 35.3 & 7.8 & 7.5 & 11.0 \\
\hline
\end{tabular}


value. Such an agreement is also illustrated by the data shown in Fig. 3. As follows from the results presented in Table 4, the main contribution to the one-neutron decay of the GDR in a few medium-heavy neutron closed-shell nuclei gives the statistical decay. Only for ${ }^{48} \mathrm{Ca}$, the direct decay contribution is markable.

\section{Conclusive remarks}

In the present work, we implement the newly developed particle-hole dispersive optical model to evaluate the partial and total branching ratios for direct one-neutron decay of the isovector giant dipole resonance in a few neutron closed-shell nuclei. The single experimental result, concerned with the total branching ratio for direct one-neutron decay of the mentioned resonance in ${ }^{48} \mathrm{Ca}$, is satisfactorily described. The next step in this study is supposed to be a description of the differential partial "direct + semi-direct" $(\gamma, n)$ - and $(n, \gamma)$ - reaction cross sections in a vicinity of the giant dipole resonance, bearing in mind an improvement of the previous study of Ref. [2].

This work was partially supported by the Academic Excellence Project of the National Research Nuclear University "MEPhI" under contract by the Ministry of Education and Science of the Russian Federation No 02.A03.21.0005 (M.U.).

\section{References}

[1] M. Urin, Phys. Rev. C 87, 044330 (2013)

[2] B. Tulupov and M. Urin, Phys. Rev. C 90, 034613 (2014)

[3] M. Gorelik, S. Shlomo, B. Tulupov, and M. Urin, Nucl. Phys. A 970, 353 (2018)

[4] S. Shlomo, G. Bertsch, Nucl. Phys. A 243, 507 (1975)

[5] M. Urin, Nucl. Phys. A 811, 107 (2000)

[6] M. Gorelik, S. Shlomo, B. Tulupov, and M. Urin, Nucl. Phys. A 955, 116 (2016)

[7] G. Kolomiytsev, S. Igashov, and M. Urin, Phys. At. Nucl. 77, 1105 (2014)

[8] G. O'Keefe, M. Thompson et al., Nucl. Phys. A 469, 239 (1987)

[9] I. Boboshin et al., CDFE nuclear data bases, http://depni.npi.msu.su/cdfe

[10] A. Veyssiere, H. Beil, R. Bergère, P. Carlos, and A. Lepretre, Nucl. Phys. A 159, 561 (1970)

[11] S. Strauch, P. von Neumann-Cosel, A. Richter, C. Rangacharyulu, G. Schrieder, K. Schweda, and J. Wambach, Phys. Rev. Lett. 85, 2913 (2000) 